Bull. Geol. Soc. Finland 41, 183-192 (1969)

\title{
ZWEI NEUE FUNDORTE DER KARBONATKONKRETIONEN IM ERDBODEN SÜDFINNLANDS
}

\author{
GunNar GlüCKert \\ Quartärgeologisches Institut der Universität zu Turku, Finnland.
}

\begin{abstract}
Two finds of calcareous concretions, precipitated in unhardened late-Glacial soil, has been made in S- and SWFinland. Various types of so called Imatra stones, rich in carbonates, clayey and tilly in composition and occuring in varved clay and rock surface are described in this article. The $\mathrm{Ca}$ values of the concretions are high and a slight $\mathrm{Mg}$ content is to be observed in the studied samples. The high percentages of $\mathrm{Ca}$ in the stones would indicate limestone deposits in the near bedrock and an abundance of carbonates in the groundwater and local soil during the precipitation of the concretions.
\end{abstract}

\section{Einleitung}

Schon im Jahre 1851 wurden einige im Abflusstal der Stromschnelle bei Imatra entdeckte Konkretionen von Kutorga (S. 85) beschrieben. Elf Jahre später erklärte Pusirewsky (1862) die im Bänderton entstandenen harten Knollen als »Marlekor». Ausserhalb der Grenzen Finnlands haben sich u.a. Holm (1888), Westergård (1934), Tarr (1935) und zuletzt Groetzner (1868) mit dem bis jetzt noch etwas unklaren, endgültigen Vorgang der Genese verschiedener Art Kalkknollengebilde sowohl im unverfestigten Erdboden als im Sandstein und Schiefer beschäftigt. Die im Ton gefundenen Tonverhärtungen sind mit den im Schiefer entdeckten, Knollengebilden analogisch, deren Zusammensetzung gleichartig mit der der Imatra-Steine ist (Eskola 1932, S. 22 -23). In Finnland sind die Imatra-Steine früher u.a. von Moberg (1884, 1885, 1888), Laitakari (1929), Eskola (1932), Salmi (1959) und Virkku-

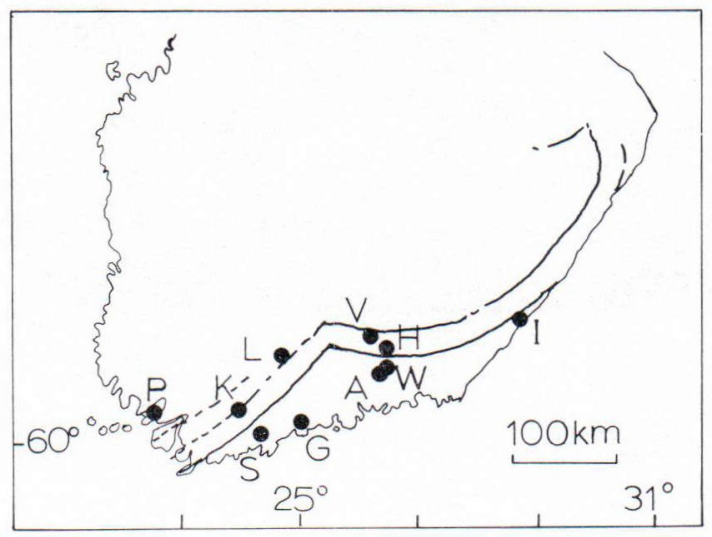

Abb. 1. Verbreitung der bisher entdeckten Karbonatknollenfunde (Punkte) im Erdboden Finnlands. Erklärung der Buchstaben: I = die Stromschnelle bei Imatra (Kutorga 1851), $\mathrm{A}=$ Pyhäjärvi, Artjärvi (Moberg 1884), $\mathrm{W}=$ Willikkalanjärvi, Artjärvi (Moberg 1885), H = Titti, V = Vuolenkoski, Iitti (Salmi 1959), L = Leppäkoski (Eskola 1932), $\mathrm{G}=$ Gammelstad, Helsinki (Moberg 1885), Kirkkojoki, Siuntio (Virkkunen 1966), K = Koisjärvi, Pusula (Glïckert 1968 a) und P = Parainen (Glückert 1968 b). nen (1966) untersucht worden (Abb. 1). 
Zweck der vorliegenden Untersuchung ist es die zwei im Südteil des Landes neulich entdeckten Knollenfunde darzulegen: die Tonkarbonatknollen im unverfestigten Ton aus Koisjärvi und die Sandkarbonatknollen aus Parainen, die auf Felsen unter Moräne entstanden sind (Glückert 1968 a und $\mathrm{b})$.

In Koisjärvi, Kirchspiel Pusula, Südfinnland, fanden einige Brunnengräber Anfang Februar 1968 aus einem 13 m tiefen Brunnen hellgraue, betonartige Verhärtungen in einer Tiefe von 9$11 \mathrm{~m}$ im schwach gebänderten Glazialton mit dünnen grobkörnigen Feinsandschichten eingebettet. Die Fundstelle liegt im Walde gegenüber der Benzinstation bei der Wegscheide nach Somero an der Hauptverkehrstrasse $58 \mathrm{~km}$ von Helsinki nach Turku. Die absolute Höhe der Erdoberfläche beim Brunnen beträgt $42 \mathrm{~m}$. Ausserdem wurden zahlreiche Steine und kleinere Stücke unter den Knollen gefunden.

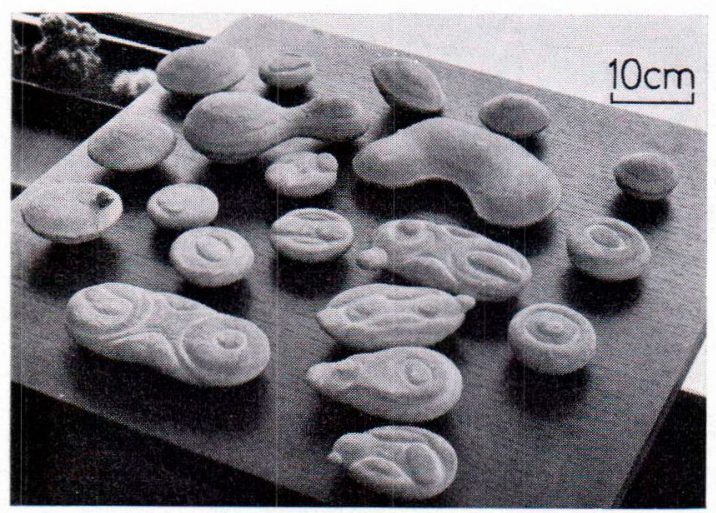

Abb. 2. Verschiedene Typen der Konkretionen von Koisjärvi. Photo V. Salenius.

In Parainen, Südwestfinnland, entdeckte der Verfasser dieses Artikels im Mai 1968 auf einigen entblössten Rundhöckern (8-10 m. ü.d.M.) mit glazialen Schrammen unter einer 4-5 m mächtigen, neulich beseitigten Moränenschicht Hunderte von ausnahmsweise kleinen, platten Kalkkonkretionen mit bräunlichgrauer Farbe. Auf den glatt polierten Felsen (Migmatit und Kinzigitengneis), die in unmittelbarer Nähe der west- lichen Steilwand der Kalkgrube von Limberg, Paraisten Kalkkivuori AG liegen, sassen die winzigen zementierten Knollengebilde nicht besonders fest, denn sie konnten mit einem leichten Hammerhieb losgehauen werden.

\section{Form und Grösse der Knollen}

In Gestalt und Grösse variieren die Knollengebilde mit zahlreichen Zwischenformen im erheblichen Masse und mehrere sowohl kennzeichnende Züge als merkbare Unterschiede sind wahrzunehmen. Morphologisch gleichen die zementierten Tonverhärtungen von Koisjärvi

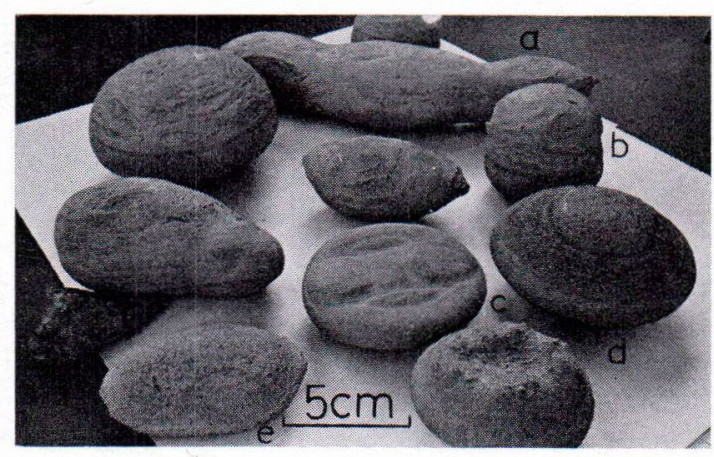

Abb. 3. Wurstförmige (a), sphärische (b), linsenförmige (c, d) und elliptische Konkretionen (e) aus Koisjärvi (von oben betrachtet).

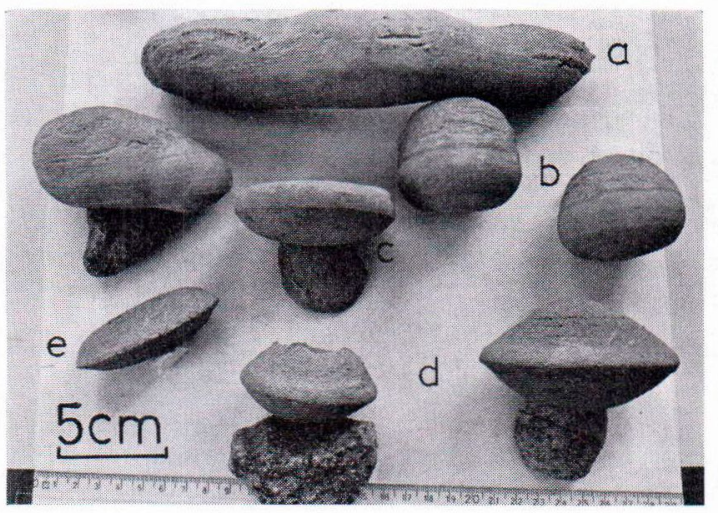

Abb. 4. Gebänderte Konkretionen aus Koisjärvi von der Seite betrachtet: wurstförmiger Typ (a), annähernd sphä rische Typen (b), Halblinsentyp mit Figuren (c), linsenförmige Typen (d), Ellipsentyp (e) sowie im Glazialton gefundene kantige Steine (vgl. Abb. 3). 
einigermassen den Imatra-Steinen von Vuolenkoski (Salmi 1959, S. 16-21). Die erstgenannten vertreten walzen-, scheiben-, linsen- und ellipsenförmige sowie sphärische Körper, aber auch längliche Konkretionen des Doppelkugeltyps mit wechselnd intensiver Verschmelzung der beiden, selten gleichgrossen Teilelemente kommen vor. Ausserdem sind die Gebilde im Glazialton von Koisjärvi auch oft ziemlich lang und wurstförmig (Abb. 2, 3, 4 und 5).

Am häufigsten treten runde, im Querschnitt ein bisschen platte Linsenformen auf, von denen mehrere mit scharfer Äquatorialkante versehen sind. Der Durchmesser der Konkretionen beträgt im Mittel 2-5 cm, während die Länge zwischen $5-20 \mathrm{~cm}$ wechselt. Die glatte Oberseite der Koisjärvi-Knollen erweist sich als flachgewölbt oder beinahe eben, die rauhere untere Seite ist dagegen oft mehr gewölbt. Einige besitzen festgewachsene kleine Steine und Sandkörner oder nur eine vom Stein eingedrückte Vertiefung.

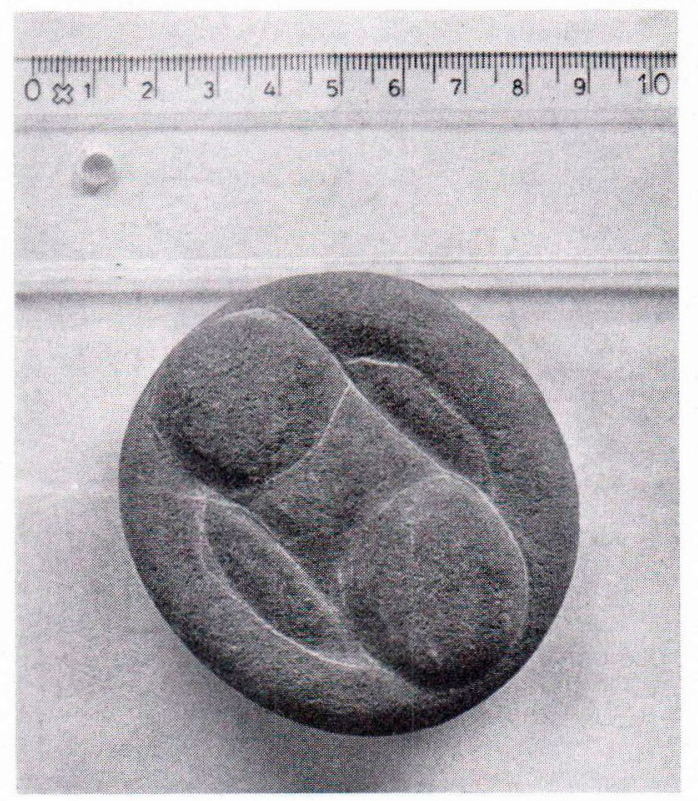

Abb. 5. Runder Knollen mit länglichem, halblinsenförmigem Querschnitt (vgl. Abb. 4, c) aus mehreren kleinen Konkretionsanfängen in verschiedenen Entwicklungsstadien zusammengewachsen.
Am allerauffälligsten aber sind ohne weiteres die rund-länglichen, flach-scheibenförmigen Knollenkörper mit kreis- und ellipsenförmigen Figuren auf der ziemlich ebenen Oberseite (Abb. 2 und 5.), als seien sie gezeichnete Eindrücke eines steinzeitlichen künstlerischen Urmenschen. Die kreis- und ovalenförmigen Figuren verlaufen auf der Knollenoberfläche in annähernd gleichem Abstand vom Rand. Ähnliche Sonderfiguren sind im Finnland bisher nur im Bänderton auf Kalkkonkretionen von Siuntio aufgefunden worden (Virkkunen 1966, S. 118-120).

Die Konkretionen lagen in Koisjärvi in einer Tiefe von rund 9-11 $\mathrm{m}$. In den aufeinander folgenden Tonschichten mit schmalen Feinsandbändern traten zuunterst vorzugsweise beinahe sphärische und längliche bis wurstförmige Körper mit kantigen Stein- und Blockstücken auf. In einer $10 \mathrm{~m}$ tiefen Knollenschicht wurden die meisten mit Figuren versehenen Gebilden entdeckt. Aus $9 \mathrm{~m}$ Tiefe kamen vorwiegend platte, linsenförmige und zweiteilige Körper, alle mit scharfer Äquatorialkante ausgestattet zum Vorschein.

Bezeichnend für die Gestalt der am Felsen festsitzenden, nichtsphärischen Knollengebilde von Parainen sind $1-2 \mathrm{~cm}$ dicke, platte Halbkugeltypen von $1-5 \mathrm{~cm}$ Länge, wie auch sehr flach ausgebildete (nur $0.3-1 \mathrm{~cm}$ im Durchschnitt) und längliche $(5-30 \mathrm{~cm})$ Streifen (vgl. Westergård 1934, S. 13-14 und Kers 1964, S. 286-287). Diese zuletzterwähnten, hin und her laufenden, unregelmässig geförmten Konkretionsflecken, die die glatte Rundhöckeroberfläche teilweise bedecken, verleihen dem Felsen ein gestreiftes, unreines Aussehen. Die Unterseite aller Knollenkörper ist stets eben, da sie harte Felsen als Unterlage haben. Die Verhärtungsgebilde an den Felsen in Parainen können in vieler Hinsicht mit den an Steinblöcken festgewachsenen Knollen im Glazialton von Vuolenkoski (Salmi 1959, S. 18-19) verglichen werden. 


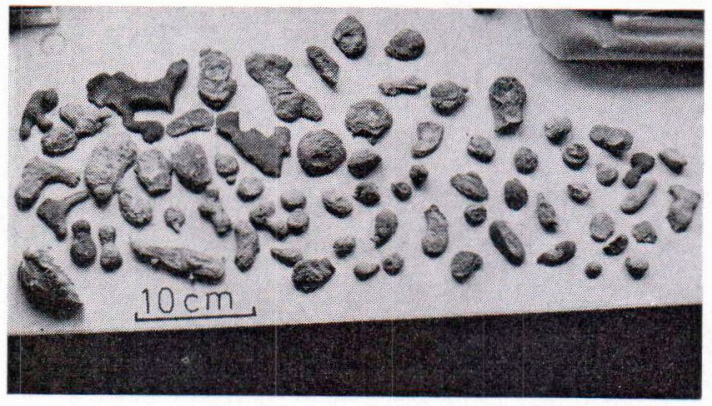

Abb. 6. Verschiedenförmige Knollentypen von Parainen. Photo M. Kokkola.

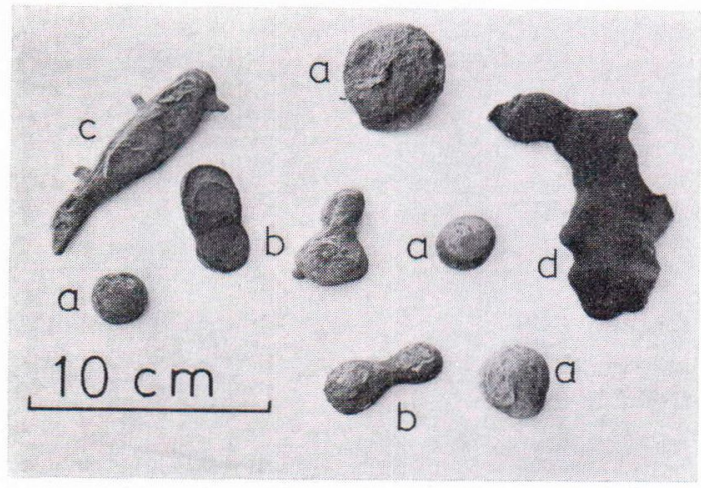

Abb. 7. Knollentypen mit halbkugelförmigem Querschnitt von Parainen: runde Typen (a), Doppelkugeltypen (b), länglicher Typ mit Ausläufern (c) und unregelmässiger Fleckentyp (d). Photo M. Kokkola.

Doppelkugeltypen mit gleich grossen Einzelelementen, etwas platte, rundlich gewölbte Formen sowie längliche Knollen, alle mit halbkugelförmigem Querschnitt kommen besonders an den steiler abfallenden Flanken der Rundhöcker vor (Abb. 6 und 7.). Die offensichtlich unbestimmt geförmten Flecken der dünnschichtigen Knollenstreifen sehen etwas sonderbar aus. Die graue Knollenmasse verunreinigt den polierten Rundhöcker und er sieht aus, als hätte jemand Schmutz, der dann härter geworden wäre, auf die sonst reine Felsenfläche gespritzt. Derartige Knollengebilde, die man bisher in Finnland nicht angetroffen hat, laufen meist lediglich auf der horizontal gelegenen oder sehr sanft neigenden, vom Inlandeis gehobelten Felsenoberseite ungefähr in der Richtung der Schrammen hin (Abb. 8 und 9).

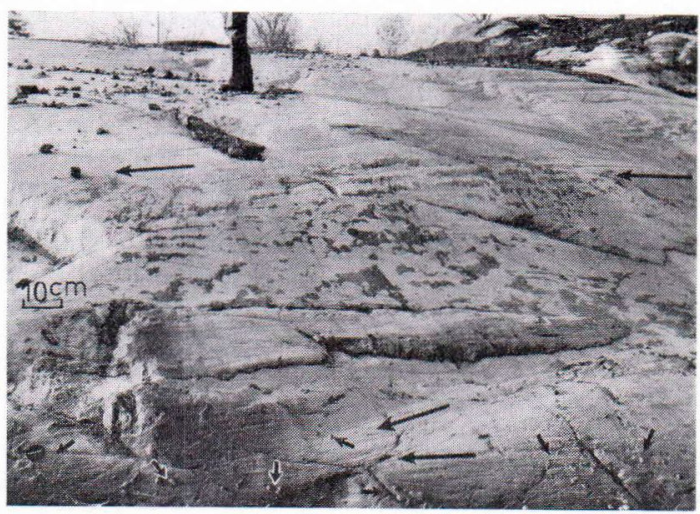

Abb. 8. Knollenflecken am Felsen unter beseitigter Moränenschicht in Parainen. Lange Pfeile = glaziale Schrammen, kurze Pfeile = winzige halbkugelförmige Knollen an den steil abfallenden Flanken des Rundhöckers.

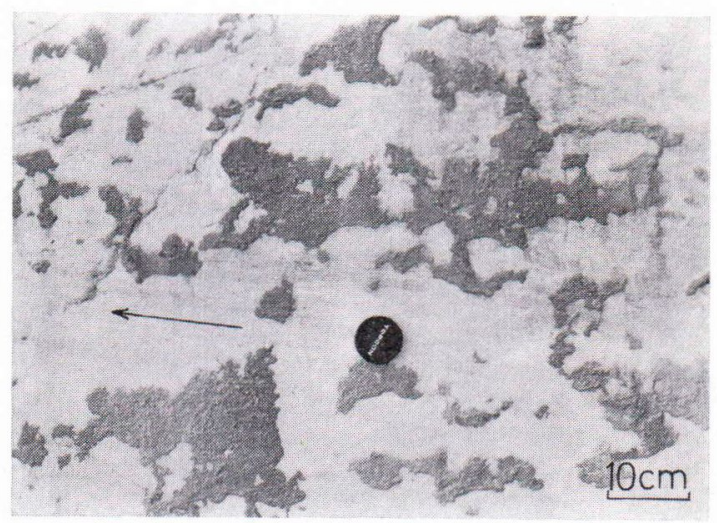

Abb. 9. Fleckenförmige Konkretionsmasse auf der Felsenoberfläche in Parainen. Pfeil = Richtung der glazialen Schrammen.

\section{Einteilung der Knollentypen}

Die bisher in Finnland entdeckten Knollengebilde sind der Form nach sehr vielgestaltig. Jede neue Fundstelle weist auch morphologisch neue Knollentypen auf, und ihr Bau und ihre Ober fläche haben jedesmal anders ausgesehen. Als Quellen dieser morphologischen Typeneinteilung 
sind einige früher herausgegebene Untersuchungen hauptsächlich von Salmi (1959, S. 16-21), Virkkunen (1966, S. 118-120) und Groetzner (1968, S. $221-223$ ) benutzt worden.

In der vorliegenden Arbeit werden zwei verschiedene Typeneinteilungen der Konkretionen vorgelegt, die sich meistens auf die Funde von Vuolenkoski, Siuntio, Koisjärvi und Parainen gründen. Die erstere Teilung (I) ist vereinfacht und stützt sich auf die morphologischen Haupttypen der Knollen, welche von zwei einander vertikalen Richtungen, von »oben» und von der »Seite» her betrachtet werden, wenn der Körper auf den Tisch in »seine natürliche Lage» gestellt ist (vgl. Abb. 3 und 4.)

Dem Obenerwähnten gemäss können die Konkretionen in acht Haupttypen geteilt werden, die folgend lauten (Abb. 10, I.):

\section{(I) 1. Sphärischer Typ (rund)}

2. Walzenförmiger Typ (länglich)

3. Ellipsenförmiger Typ (länglich)

4. Linsenförmiger Typ (rund und länglich)

5. Scheibenförmiger Typ (rund und länglich)

6. Doppelkugeltyp (länglich)

7. Halbkugeltyp (rund und länglich)

8. Unregelmässiger Typ (länglich)

Die meisten Formen sind irgendwie orientiert, denn runde Körper kommen nur bei den sphärischen, linsen- und scheibenförmigen sowie halbkugelförmigen Typen vor. Kurze Walzen gehen oft in längere wurstförmige Formen über. Die linsen- und scheibenförmigen Typen haben bisweilen Figuren und schwierig teilbare Zwischengruppen kommen natürlich relativ üblich bei allen Gruppen vor. Die sowohl halbkugelförmigen als unregelmässigen, aus dünner Knollenmasse bestehenen Flecken und Streifen mit unzähligen Kombinationen und Variationen sieht man auf Felsen, und viele von ihnen sind mit Steinen und Blockstücken festgewachsen. Die letzte Gruppe der Typeneinteilung umfasst somit unzählige Variationen und dient auch als Notbehelf der komplizierten Fälle.
Eine ausführlichere Typeneinteilung (II) erfordert genauere Begründungen. Man muss wissen aus wievielen (eine, zwei oder drei) Richtungen man den Knollen betrachtet und welch eine Form die Quer- und Längsschnitte des Knollenkörpers besitzt. Alle Konkretionen sind im Prinzip rundlich an den Ecken und dreidimensional und können demnach in drei rechtwinkelige Achsenflächen geteilt werden. Der kürzeste Durchschnitt, der Querschnitt der Körper, ist rundförmig dann, wenn die zwei anderen, zugleich längeren Durchschnitte, die Längsschnitte gleichförmig, symmetrisch sind. Deswegen werden solche Knollen als Typen mit rundem Querschnitt (1) genannt und von der länglichen Seite, z.B. von oben und vom runden Ende betrachtet.

Wenn aber alle rechtwinkeligen Achsenflächen verschiedenförmig sind (eine Fläche kann trotzdem rund sein), sehen die Knollen von allen Seiten verschieden aus. Solche Körper gehören zu dem mit plattem Längsschnitt versehenen Typ (2). Sie werden auf dem Tisch länglich plattgestellt und von oben und von einer, der längsten platten Seite her, betrachtet. Alle Knollentypen werden bei dieser vielseitigeren Typeneinteilung nur von zwei einander rechtwinkeligen Richtungen gemustert, also von oben und vom Ende oder einer der längeren Seite.

Nach solcher Betrachtung der Knollentypen erhalten wir nebst zahlreichen Unterformen drei verschiedene Typengruppen (Abb. 10, II.):

(II) 1. Die Formentypen mit rundem Querschnitt,

2. Die Formentypen mit plattem Längsschnitt,

3. Die Formentypen mit halbkugelförmigem Längsschnitt.

\section{Typen mit rundem Querschnitt}

1.1 Sphärischer Typ

1.2 Länglicher Typ

1.21 Walzenförmiger Typ (kurz, wurstförmig; gerade und gebogen) 


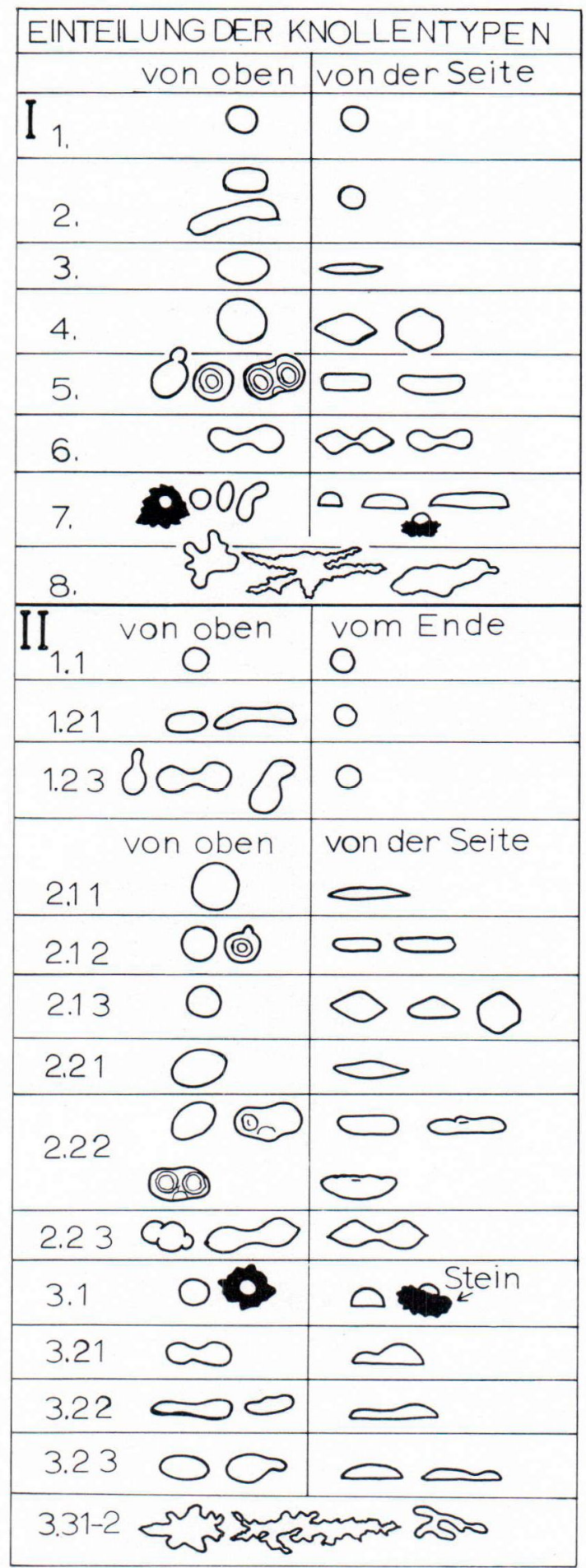

Abb. 10. Die Einteilungen I und II der Knollentypen.
1.22 Doppelkugeltyp (gerade und gebogen; mit gleich-und ungleichgrossen Teilelementen)

\section{Typen mit plattem Längsschnitt}

2.1 Runder Typ

2.11 Elliptischer Typ

2.12 Scheibenförmiger (Münzenförmiger) Typ (bisweilen mit Figuren; Siuntio)

2.13 Linsen- und halblinsenförmiger Typ (bisweilen mit Figuren; Koisjärvi)

2.2 Länglicher Typ

2.21 Elliptischer Typ

2.22 Scheibenförmiger und halblinsenförmiger Typ (ohne und mit Ausläufern und Figuren; Siuntio, Koisjärvi)

2.23 Doppelkugeltyp (mit gleich- und ungleichgrossen Teilelementen)

\section{Typen mit balbkugelförmigem Längsschnitt}

3.1 Runder Typ

3.2 Länglicher Typ

3.21 Doppelhalbkugeltyp (ohne und mit Ausläufern und mit gleich- und ungleichgrossen Teilelementen)

3.22 Wurstförmiger Typ

3.23 Scheibenförmiger Typ (ohne und mit Ausläufern und Figuren; Siuntio)

3.3 Unregelmässige Formentypen

3.31 Flecken (Parainen)

3.32 Streichen (Parainen)

3.33 Sondertypen

\section{Bau und Material}

Das Material der kompakten, betonharten Karbonatknollen von Koisjärvi besteht aus gut klassiertem quartären Schmelzwasserton, wonach das tonig-karbonatische Material als Karbonat- 
ton anzusprechen ist. In den kugelähnlichen und den mit scharfer Äquatorialkante versehenen Steinen ist eine gemeinsame, bisweilen kleine Feinsandlinsen enthaltende, kontrastarme Schichtung am besten zu beobachten (Abb. 3 und 4). Die typische gebänderte Struktur der Knollenkörper ist ein Beweis für den an und für sich gehärteten, primären, geschichteten Glazialton. Gebänderten Bau haben auch die Konkretionen von Artjärvi (Moberg 1885, S. 50) und Siuntio (Virkkunen 1966, S. 118 -120). Zerschlagen zeigen die harten Steine von Koisjärvi, sowie die Imatra-Steine bei Vuolenkoski (vgl. Salmi 1959, S. 20-21), eine feinstkörnige, massive, schwachgebänderte, graufarbige Bruchfläche bisweilen mit einem Sandkorn im Knollenzentrum.

Die Messungen des Eigengewichts erweisen, dass die Knollen etwas leichter als die herrschenden Gesteinsarten des Felsengrundes am Fundorte sind. Die Bestimmungen des spezifischen Gewichts der Verhärtungen von Koisjärvi betragen 2.58-2.63, rund 2.61, die von Parainen 2.54 -2.62, rd. 2.58 und zum Vergleich die von Vuolenkoski $2.44-2.63$, rd. 2.53 .

Die etwas weniger harten, oft rauh körnigen, ungeschichteten Konkretionen auf der Felsenfläche in Parainen haben ihr Baumaterial von der ihr überliegenden, sandigen Moränenschicht erhalten. Die als Bindestoffe der Mineralkörper dienenden Karbonate stammen aus den in unmittelbarer Nähe liegenden Kalksteinvorkommen und sind mit kalkhaltigem Grundwasser auf der gestreiften Felsenoberfläche herbeigeführt worden.

Die in Formentypen ausserordentlich variierenden Knollenkörper von Parainen sind aus quarzreicher, feldspatarmer Feinsandmoräne aufgebaut, die durch karbonatisches Bindemittel zusammengehalten werden. Beim Auflösen der Sandkonkretionen in $\mathrm{HCl}$ bestand der unlösbare Rest durch ein Stereomikroskop betrachtet überwiegend (99\%) aus spärlich gerundeten Quarzkörnern. Sowohl der Feldspatenanteil als die kantigen Quarzkörner mit karbonat-gefüllten, unregelmässigen, winzigen Aushöhlungen weisen auf Korrosionvorgänge hin (Groetzner 1968, S. 224). Ausser Quarz und Feldspäte sind im Lösungsrückstand noch Einzelkörner von Glimmer und Hornblende übriggeblieben.

\section{Karbonatgehalt der Konkretionen}

Um die chemischen $\mathrm{Ca}$ - und $\mathrm{Mg}$-Gehalte zu untersuchen wurden die Experimente der Proben mit Heranziehung von zwci Methoden durchgeführt. Das Karbonat der Knollen und der dicht um sie herum liegenden Ton- und Moränenschichten wurde nach der Lösung mit Salzsäure (gekocht) aus dem Gewichtsverlust errechnet und nachträglich mit dem Perkin-Elmer (Atomic absorption spectrophotometer Perkin-Elmer 290) aus verdünnter $(1: 200) \mathrm{HCl}$-haltiger Lösung kontrolliert. Beim Vergleich der Zahlenwerte der beiden Methoden erwies sich die Tatsache, dass das durch Auflösen mit Salzsäure ermittelte, durch Gewichtsverlust erhaltene Resultat nur sehr wenig von dem des Perkin-Elmers abwich. Somit besteht das Karbonat der Konkretionen hauptsächlich nur aus $\mathrm{Mg}$ und $\mathrm{Ca}$.

Das Mischkarbonat der untersuchten Proben bestand vorwiegend aus Calcit, denn der Anteil des Magnesiums (Dolomit?) war gering. Die einfache Salzsäurenmethode ergab den Totalgehalt des Karbonats mit einer Genauigkeit von $1-3$ $\%$, aber mit dem Perkin-Elmer wurden beträchtlich exaktere Zahlenwerte des $\mathrm{Ca}$ - und $\mathrm{Mg}-\mathrm{Ge}$ halts ermittelt. Das $\mathrm{Ca} / \mathrm{Mg}-$ Verhältnis liegt zwischen 13 und 33 (Konkretionen), 8 und 10 (Moräne) und zwischen 0.6 und 0.9 (Ton). Die Ca-Menge schwankt in den Proben aus Koisjärvi zwischen 15-22\% (Konkretionen) und $0.8-0.9 \%$ (Ton), in den Proben aus Vuolenkoski zwischen 22-24\% $(\mathrm{CaO})$ und 2.2 $2.5 \%$ ( $\mathrm{CaO}$, Ton). Der Mg-Anteil bleibt stets unter $1.7 \%$ (Abb. 11.). Bemerkenswert ist die hoche Ca-Menge der Moräne (3.8-4.0\%) mit der kleinen $\mathrm{Ca}$-Menge des Tons $(0.90 \%)$ verglichen, während der Prozentwert des $\mathrm{Mg}$ höher im Ton (0.92-1.61) als in der Moräne (0.40$0.45)$ liegt. 
Tabelle 1.

Ca-, Mg- und Karbonatbestimmungen an Kalkkonkretionen, Ton und Moräne von Parainen und Koisjärvi mit den Resultaten früherer Untersuchungen verglichen: 1) Salmi 1959, 2) Moberg 1884, 3) Moberg 1885, 4) Holm 1888, 5) Eskola 1932 und 6) Westergård 1934. $\mathrm{K}=$ Konkretion, $\mathrm{T}=$ Ton, $\mathrm{M}=$ Moräne. Die $\mathrm{Mg}-\mathrm{und} \mathrm{Ca}-$ Prozentwerte sind mit dem Perkin-Elmer (Atomic absorption spectrophotometer Perkin-Elmer 290) bestimmt, die Oxyd- und Karbonatwerte $(\%)$ sowie $\mathrm{Ca} / \mathrm{Mg}$ sind berechnet.

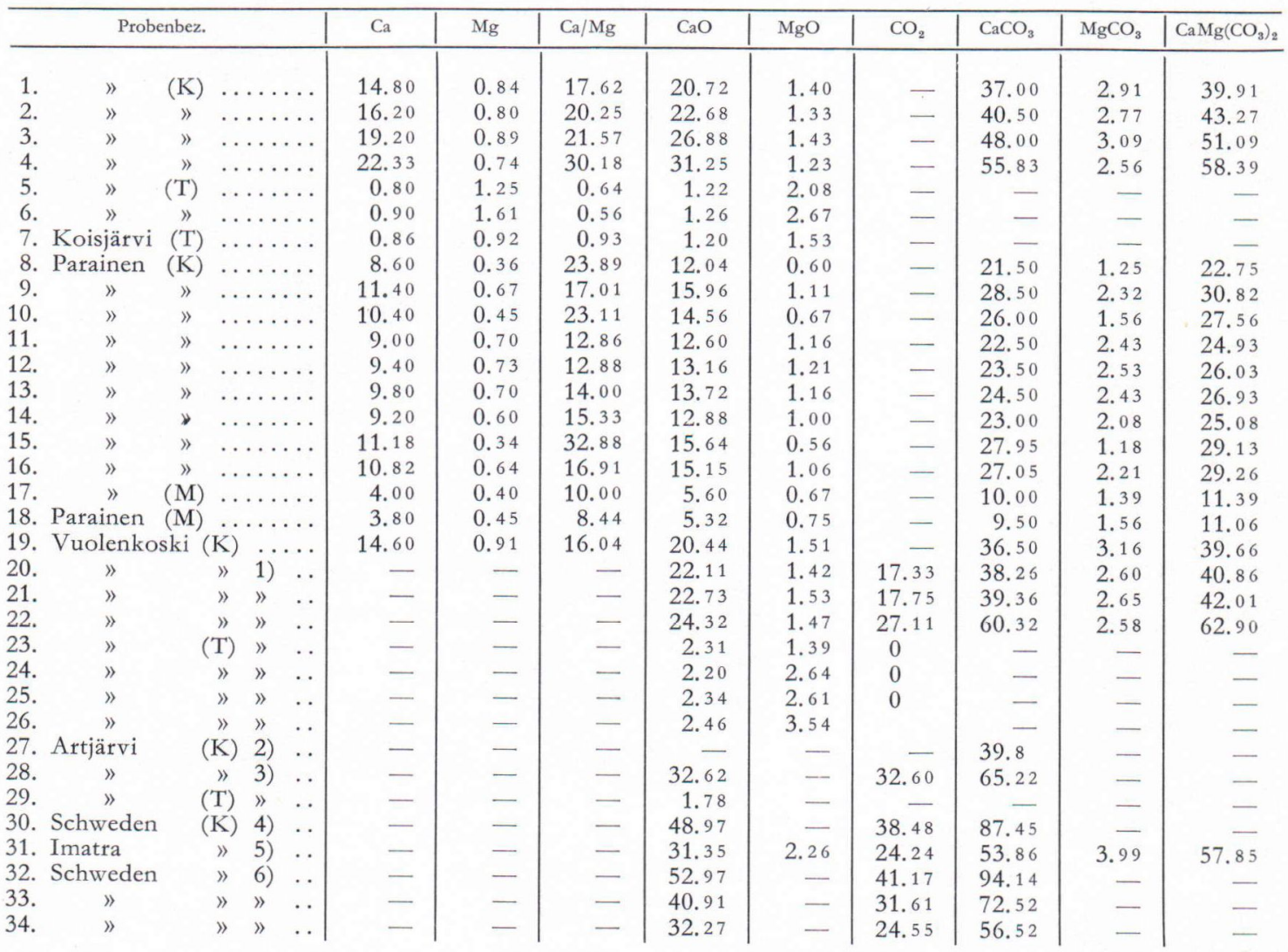

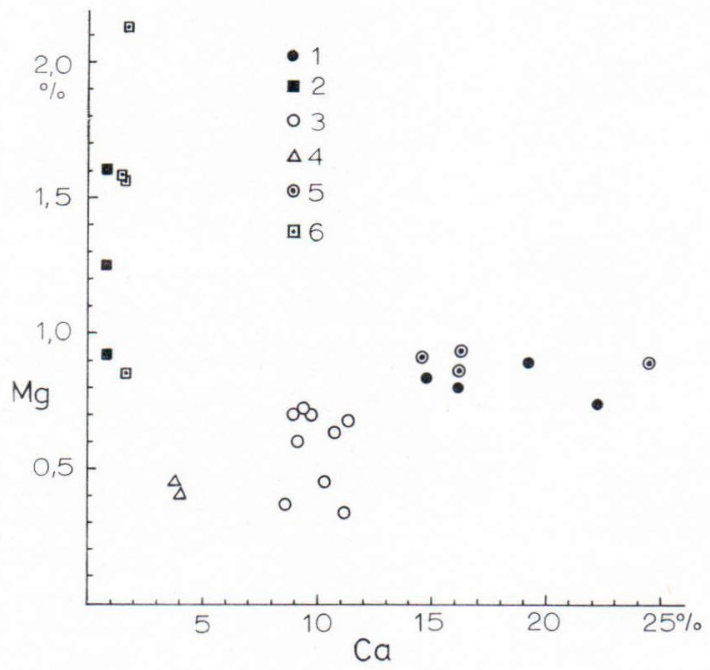

Abb. 11. Das Ca- und Mg-Verhältnis derKnollen-, Tonund Moränenproben. $1=$ Konkretion, Koisjärvi, $2=$ Ton, Koisjärvi, $3=$ Konkretion, Parainen, $4=$ Moräne, Parainen, 5 = Konkretion, Vuolenkoski (aus Salmi 1959, berechnet) und $6=$ Ton, Vuolenkoski (aus Salmi 1959, berechnet). 
Die Auswertung von den chemischen $\mathrm{Ca} / \mathrm{Mg}$ Analysen der 19. Proben brachten verschiedene Ergebnisse für die Karbonatmengen, die mit den Resultaten früher untersuchten Proben von Moberg (1884, 1885), Holm (1888), Eskola (1932), Westergård (1934) und Salmi (1959) verglichen werden (Tabelle 1). In den Tonschichten von Koisjärvi ist $\mathrm{CaO}$ ziemlich gleichmässig verteilt und mit niedrigem Prozentgehalt vorhanden (1.12-1.26). In den Konkretionen dagegen ist die Karbonatmenge bedeutend höher, indem sie zwischen 40 und $58 \%$ (rd. 21-31 \% CaO) variiert. Bei den Kalkverhärtungen von Parainen sind die Schwankungen etwas kleiner, rd. 23$31 \% \mathrm{CaMg}\left(\mathrm{CO}_{3}\right)_{2}$. Die Zahlenangaben der Konkretionen von Vuolenkoski übertreffen (40 -63\% $\left.\mathrm{CaMg}\left(\mathrm{CO}_{3}\right)_{2}\right)$ auch die Resultate der in Amerika gemachten Untersuchungen, indem die Karbonatwerte jenseits des Atlantiks nach Tarr (1935) zwischen 35 und $53 \%$ variieren.

\section{Zur Genese der Konkretionen}

Das mit der Anziehungskraft eines Knollenzentrums folgende Entstehen der Konkretionen und ihr Entwicklungsverlauf dürfte nicht besonders kompliziert sein. Den Hauptteil des Bindestoffes zwischen den erkennbaren Komponenten bilden Karbonate. Das als Bindemittel dienende karbonatische Zwischenmaterial füllt die Porenraume der Mineralkörper (Groetzner 1968, S. 224). Die Konkretionen sind durch karbonatreiches Bindematerial aus jeweilig zur Verfügung gestelltem Material auf der gleichen Stelle während einer langen Zeit allmälich um ein Karbonatfällungszentrum mit Zugkraft (z.B. ein Karbonatkorn, Sandkorn, Steinfragment oder Felsenoberfläche) chemisch ausgefallen. Dazu hat wohl die vielleicht durch die eiszeitliche Einanderreibung der Zentrenkörper entstandene elektrische Anziehungskraft beigetragen.

Die waagerechte Lage der Knollenschichtung von Koisjärvi beweist, dass die Konkretionen sich im unverfestigten Erdboden aus dem Tonmaterial auf derselben Stelle nach und nach gebildet haben. Somit ist die Bänderung der ursprünglichen Tonlagen in den härtenden und zugleich in Grösse wachsenden Konkretionen mitgenommen worden und an und für sich wahrnehmbar erhalten (Abb. 3 und 4.).

Die originalen, rundlichen Figuren auf der waagerechten Oberfläche der platten KoisjärviVerhärtungen deuten auf Stellungen der Ränder von gradweisen Zuwachsstadien der kleineren Knollenscheiben im Bänderton hin. Besonders sind die rundförmigen Knollen langsam in alle Richtungen gewachsen, bei länglichen Formen ist der Wuchs aber meist in waagerechter Richtung den Schichtflächen folgend zu beobachten. Ebenfalls können zwei- und dreiteilige Mehrkugelkombinationen mit verschiedenen $Z_{w i-}$ schenformen als Produkte konkurrierender, einander sehr nahe, in derselben Horizontalebene liegenden Fällungszentren und winzigen Knollenanfängen auftreten.

Das Gefüge im Zusammenhang mit dem Nebengestein zeigt, dass die Blöcke und der Felsen als knollenbildende Unterlage dienen. Die vielförmigen Konkretionsflecken auf der Oberfläche der Rundhöcker in Parainen sind unter einer dicken Erdschicht der geschrammten Unterlage gemäss gegen den harten Felsen plattgedrückt worden und haben deswegen nicht in die Höhe wachsen können. An den mehr abfallenden Rundhöckerflanken, wo das Gewicht der Erdmassen auf der Felsenfläche anders geteilt ist, haben schon runde, dickere Knollen mit halbkugelgeformtem Querschnitt zementieren können (Abb. 8 und 9.).

Das reichliche Vorhandensein des Karbonatgehalts in den Konkretionen führenden Bändertonschichten zeigt auf mehr oder weniger lokal gelegene Kalksteinvorkommen im Felsengrund der Umgebung von Koisjärvi hin (vgl. Salmi 1959, S. 24). Wahrscheinlich gibt es in den Umgebungen von Kalksteinvorkommen, wie z.B. in den Gegenden von Lappeenranta und Lohja, in der Tiefe der Tonerdböden unentdeckte Vorkommen der Karbonatkonkretionen, wo es möglicherweise Knollen neuer Formentypen in grossen Mengen gibt. 
In glazialen Verhältnissen nicht weit vom Rande des Inlandeises, vor annähernd 1000012000 Jahren, wo der Ton im gelinde karbonathaltigen Schmeizwasser sedimentiert ist, hat sich der Karbonatstoff gleichzeitig mit der lokal herumliegenden Tonerde abgelagert. Von einem Konzentrationszentrum herbeigezogen haben die Karbonate zu einem langsamen, zementierenden Verknollungsvorgang in der unaufhörlich zuwachsenden Sedimentdecke als ausfallender, härtender Porenraumfüllung beigetragen.
- Dem Vorstand des Quartärgeologischen Instituts der Universität zu Turku, Herrn Dr. Professor Martti Salmi möchte ich meinen besten Dank, für die Erlaubnis das Material aus Vuolenkoski zu benutzen, für seine wertvollen Hinweise und für sein kritisches Durchgehen des Manuskripts, zum Ausdruck bringen. Meinen Dank richte ich auch an Herrn Dr. Professor K. J. Neuvonen, den Leiter des Geologisch-Mineralogischen Instituts der Universität zu Turku, für die Genehmigung den PerkinElmer-Apparat zu gebrauchen. Schliesslich danke ich noch Mag. Phil. Leo Grundström für die Hilfe beim Bestimmen der $\mathrm{Ca}$ - und $\mathrm{Mg}$-Werte mit dem PerkinElmer.

\section{LITERATURVERZEICHNIS}

Eskola, Pentiti (1932) Conditions during the earliest geological times as indicated by the archaean rocks. Ann. Acad. Sci. Fennicae, Ser. A, 36, 4, pp. 1-74.

GlüCKert, GunNar (1968 a) Imatrankivilöytö Pusulan Koisjärvellä. Geologi, Vuosik. 20, 2-3, p. 11.

- (1968 b) Imatrankiviä Paraisten kalkkikivialueclla. English summary: Calcareous concretions in Parainen, SW-Finland. Geologi, Vuosik. 20, 10, pp. 141-143.

Groetzner, Johann-Peter (1968) Bau und Genese von "Sandsteinkugeln» aus dem Lias $\alpha 1$ (Hettangien) von Helmstedt. Beih. Ber. Naturh. Ges. 5, Keller-Festschrift. Hannover 1968, pp. 219-234.

HoLm, G. (1888) Om några marlekeliga bildningar förekommande i sprickor inom alunskiffern vid Knifvinge i Vreta Kloster socken i Östergötland. Geol. Fören. Förhandl. Bd. 10, H. 2, pp. 116-122.

Kers, Lars-Erik (1964) Subglacialt utfälld kalksten i Solna samt i Garsvik, Västerbottens län. Geol. Fören. Förhandl, Bd. 86, H. 3, pp. 282-310.

Kutorga, S. (1851) Geognostische Beobachtungen im südlichen Finnland. Verh. Ges. Min. Petersburg.

Laitakari, Aarne (1929) Imatran kivistä. Uusi Suomi, 183.
Moberg, K. Ad. (1884) Beskrifvning till Kartbladet No. 7. Finl. Geol. Unders., pp. 1-87.

- (1885) Beskrifvning till Kartbladet No. 8. Finl. Geol. Unders., pp. 1-77.

Pusirewsky, P. (1862) Neue Fundorte der Morpholiten in Finnland. Verh. Ges. Min. Petersburg.

SALMI, MartTi (1959) Imatra stones in the glacial clay of Vuolenkoski. Bull. Comm. géol. Finlande, 186, pp. $1-27$.

Sauramo, Matri (1923) Studies on the Quaternary varve sediments in Southern Finland. Bull. Comm. géol. Finlande, 60, pp. 1-144.

TARR, W. A. (1935) Concretions in the Chamblain formation of the Connecticut River valley. Bull. Geol. Soc. Amer. 46, II, pp. 1493-1534.

WestergÅRD, A. H. (1934) En kvartär stromatolitkalksten från Bohuslän. Sver. Geol. Unders. Ser. C. Nr. 381, pp. $1-48$.

Virkkunen, Marjatta (1966) Kalkkikonkreetioita kerrallisessa savessa Siuntiossa. English summary: Calcareous concretions in varved clay in Siuntio. Geologi, Vuosik. 18, 8-9, pp. 118-120.

Manuskript eingegangen am 29. Januar 1969. 